Competition Among Institutions

by

Andrew Caplin, Columbia University

Barry Nalebuff, Yale University, SOM

July 1992

Discussion Paper Series No. 621 
July, 1992

\title{
COMPETITION AMONG INSTITUTIONS
}

\author{
By ANDrew Caplin and Barry Nalebuff \\ Columbia University Yale University, SOM
}

\begin{abstract}
Economic theory offers two different approaches to the analysis of group formation and the role of institutions. The general equilibrium approach explores the influence of the economic environment on the formation of groups. The game theoretic approach runs in the opposite direction; it explores the influence of institutions on economic outcomes. To integrate these approaches we consider situations in which institutions must compete for members. Our focus is on the fundamental interaction between memberships and policies. The policy that an institution adopts depends on its membership, while its membership depends on the policies of all the institutions.

We provide the basic elements for a theory of competition among institutions: an abstract definition of an institution and the corresponding equilibrium concepts. We demonstrate by example, the possibility that an equilibrium may not exist. In the absence of a general existence result, we pursue three different avenues. We begin with existence results based on maximization of a utilitarian social welfare function. This places strong restrictions on the decision-making process, although it covers a number of interesting political applications. This is followed by a continuity-based approach. Although quite general, it relies critically on an assumption that the institutions have certain idiosyncratic features. To handle cases without idiosyncrasies, we turn to an algebraic approach. Although existence is established, the result depends on the dimensionality of the problem. Together, these avenues provide a broad class of models for which equilibrium exists, covering cases with multiple dimensions, multiple institutions, and general institutional decision-making proceses.
\end{abstract}

We wish to thank Don Brown, Mike Magill, Andreu Mar-Colell, William Massey, James Munkres, and Thomas Romer for their help. This research was supported by the Deutsche Forschungsgemeinschaft, Gottfried-Wilhelm-Liebniz-Forderpreis during the authors' stay at BoWo 90 and by NSF grant \#SES91-22321. 


\section{I . INTRODUCTION}

There are two different approaches to the analysis of groups and social institutions within economic theory. The first approach, developed primarily by general equilibrium theorists, explores the influence of individual endowments, preferences, and technology on the formation of groups. Groups are seen as forming in a cooperative manner in response to efficiency needs, such as the desire to share risk or to provide a service where there are scale economies. ${ }^{1}$ While this approach clarifies the influence of the economic environment on the formation of coalitions, it downplays the influence that social institutions may exert on economic outcomes.

The second approach to institutions, developed primarily by game theorists and political scientists, takes the opposite view. An institutional structure is taken as given, and its implications for economic and political outcomes are then analyzed, typically with the tools of noncooperative game theory. Specifying the "rules of the game" is seen as a way of producing institution-specific answers to how economic and social decisions are made. ${ }^{2}$ While this approach clarifies the influence of the institution on economic outcomes, it downplays the influence of the economic environment on the structure of the institution. These models typically have only one institution to which everyone belongs, so that membership is exogenously fixed.

We believe that it is important to develop an integrated approach to institutions, one that allows both for the influence of institutions on economic outcomes and for the influence of the environment on the institutions. In this paper, we consider situations in which a set of distinct institutions compete for members. This leads to a fundamental interaction whereby the policy that each institution adopts depends on its (and others') membership, and the memberships depend upon the policies of all institutions. While institutional structure helps to shape outcomes, it is also shaped by the economic environment.

The importance of this form of competition among institutions is illustrated in the literature on local public goods inspired by Tiebout [1956]. There are several communities, each of which has a political process for deciding the provision of its local public goods based on the preferences of its population. Individuals reveal their preference for the provision of local public goods through their choice of a community. In this setting, Westhoff [1977] provides a pioneering study of the existence of equilibrium. When communities use majority rule to determine their supply of public goods and preferences are single peaked, he shows that there exists an equilibrium where each community follows the preference of its median member and each person belongs to their most-preferred

1 See, for example, Prescott and Townsend (1984).

2 See, for example, Baron and Ferejohn [1987], Austen-Smith and Banks [1988], and Baron [1991b]. 
community. Following Westhoff, there has been further work studying the existence of equilibrium in the context of local public goods. This work includes Epple, Filimon, and Romer [1984], Epple and Romer [1991], Greenberg [1983], Greenberg and Weber [1986], Richter [1982], and Rose-Ackerman [1979]. ${ }^{3}$

The problems considered in the Tiebout model are very special both in the type of institutions considered, and in the nature and dimensionality of the issue space. The analysis of institutional competition can be applied to such diverse topics as: the membership and platforms of political parties (Black [1948], Baron [1991a]); the relationship between shareholders and corporate policy; the structure of political coalitions such as the EEC (Casella and Feinstein [1990]); membership of private clubs (Buchanan [1965], Scotchmer [1983]); and self-selection into contracts (Rothschild and Stiglitz [1976]). The common thread in these examples is that they all involve situations in which there is an interaction between the membership and the actions of an institution. To cover these cases, we study institutional competition in a general setting, allowing for multidimensional choice problems, and a broad class of institutional decision-making processes.

To provide the key elements needed for a general model of competition among institutions, we begin with an abstract definition of an institution and specify two corresponding equilibrium concepts. We then illustrate by example that an equilibrium might not exist. Although there is no general existence result, we provide a brosd class of models for which equilibrium exists, covering cases with multiple dimensions, multiple institutions, and general institutional decision-making processes.

Section 2 provides an example that motivates our approach. Section 3 presents general definitions of institutions and of institutional equilibrium. Section 4 provides counterexamples to existence of equilibrium. Sections 5 and 6 develop cases in which existence can be guaranteed using standard arguments based on optimization and continuity. Section 7 explores the more challenging cases, and uses index theory to provide a result that shows a surprising role of dimensionality in ensuring existence. Section 8 offers a brief conclusion. The majority of the proofs are contained in an Appendix.

\section{II . AN EXAMPLE}

We develop a simple example that illustrates the interaction between population characteristics and the decision rules of institutions. The example is motivated by the common claim that the ability of one community to adopt an egalitarian policy may depend on whether competing communities have similarly egalitarian policies (see, e.g., Stigler[1957]). We show that adoption of an egalitarian constitution in one community

3 In addition, Richter [1982] and Greenberg [1983] consider whether s solution exists to the social planner's problem of allocating public goods and taxes acrose jurisdictions while allowing free mobility. 
may either increase or decrease the extent of inequality in society, depending not only on the policies of other communities, but also on the distribution of characteristics in the population. Since it is possible to assess this issue only in a framework where individuals choose among competing institutions, the example provides a motivation for our general approach.

There are two schools that compete for students through their choice of curricula. We describe the curriculum of each school by a single parameter, $x \in[0,1]$. For example, we might think of $x$ as representing the science content of the curriculum. Prospective students differ in their preference for $x$. A student of type $\alpha$ who attends school with curriculum $x$ has utility $-\|\alpha-x\|^{2}$, so that $x=\alpha$ is type $\alpha$ 's most-preferred school curriculum. Students pick the school based on their anticipation of which school will offer the most suitable curriculum. Since the population is large, no student believes that his or her choice of school will influence the curriculum.

Each school responds to its students through one of two different political processes. "Utilitarian" schools pick $x$ to maximize the sum of their students' utilities, while the more egalitarian "Rawlsian" schools pick $x$ to maximize the minimum utility of their student body. The political process each school adopts is taken to be part of the fixed data of the economy and is commonly understood when students decide which school to attend. In equilibrium, the population is divided between the schools in such a way that each student picks the school he or she prefers, correctly anticipating the schools' resulting curricular choices. We compare the equilibrium outcome in each of three different scenarios: the case in which both schools are utilitarian; the case in which both are Rawlsian, and the mixed case with one utilitarian and one Rawlsian school.

We first solve the model for the triangular population distribution of Figure 1. Figure 1(a) illustrates the equilibria for the pure utilitarian and the pure Rawlsian cases. In the pure utilitarian case, the unique equilibrium involves the two schools locating at $1 / 3$ and $2 / 3$ respectively. The pure Rawlsian case has the schools at $1 / 4$ and $3 / 4$ respectively. In both cases, the students divide equally between the two schools. The equilibrium in the mixed case is illustrated in Figure 1(b) for the case in which the Rawlsian school locates to the left of the utilitarian school. Here the unique equilibrium has the schools at roughly 0.2 and 0.6 , with the population to the left of 0.4 going to the Rawlsian school and the larger group to the right going to the utilitarian school."

\footnotetext{
4 Let $x$ denote the student who is indifferent between the two schools in the mixed case. Note that the Rawlaian school locates at $\bar{z}$, while the utilitarian achool locates at the center of gravity of the range $[x, 1]$, which is always closer to $x$ than to 1 , since more of the population is close to $x$ than is cloee to 1 . The unique equilibrium value of $x \in(0,1 / 2)$ is such that the utilitarian school locates at $\frac{3}{2}$, so that the student at $x$ is indeed indifferent between the schools. The precise value of $z$ is then mechanically derived as the solution to the equation $3 x / 2=\left(3-8 x^{3}\right) /\left(6-12 x^{2}\right)$.
} 

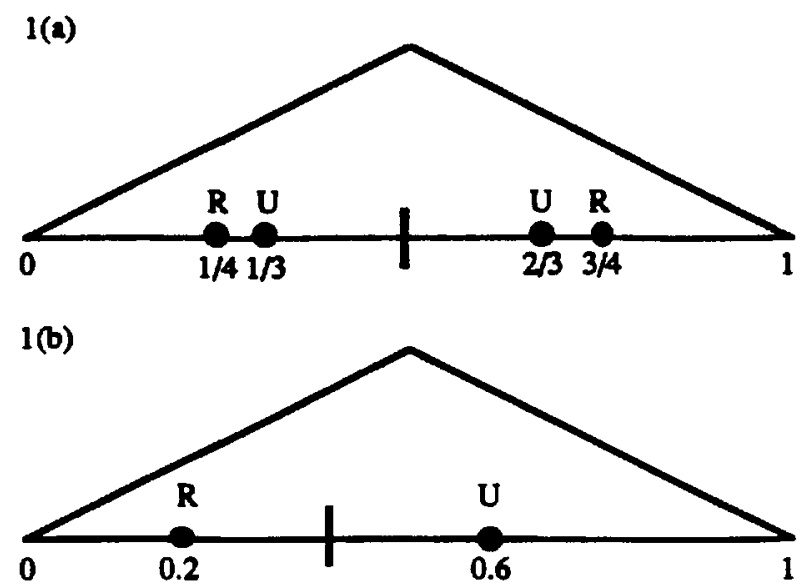

Comparing the mixed case to the pure Rawlsian illustrates the fact that the switch by one school to a more egalitarian charter may make society as a whole more egalitarian. In the mixed case, the worst-off members of society have a curriculum that is (roughly) 0.4 away from their ideal, while this maximum distance falls to 0.25 in the pure Rawlsian case. However, comparing the pure utilitarian and the mixed case illustrates that one school's switch to a more egalitarian charter may have the opposite effect; society as a whole becomes less egalitarian. In the pure utilitarian case, the worst-off member of society has a curriculum that is 0.33 away from their ideal, as opposed to 0.4 in the mixed case.

The example shows that the ability of one group to achieve an egalitarian social end can be defeated if other institutions are not similarly egalitarian. However, this conclusion is not general. To illustrate the fact that population characteristics have an important independent role in determining the nature of institutions, consider the same issue with the inverted-triangular population distribution of Figure 2. Figure 2(a) illustrates the unique equilibrium in the pure Rawlsian case, with the schools at $1 / 4$ and $3 / 4$, as well as the equilibrium for the pure utilitarian case, with the schools at $1 / 6$ and $5 / 6$. Figure 2 (b) illustrates the unique equilibrium in the mixed case, with the Rawlsian school located at roughly 0.28 and the utilitarian at $\mathbf{0 . 8 4}$, with the dividing line between the schools located at roughly 0.56 .

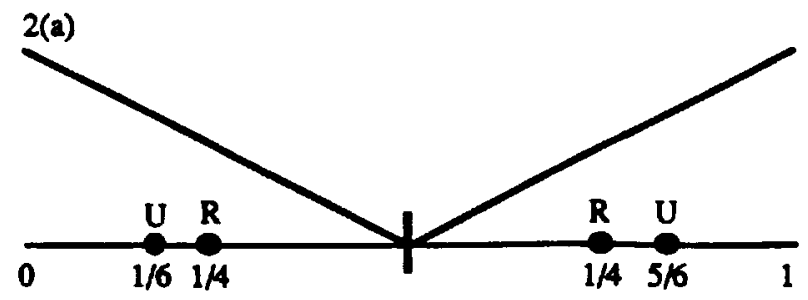




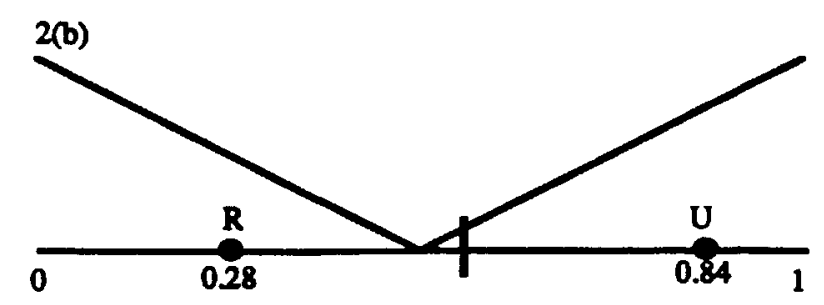

With the inverted triangular distribution, the maximum distance of any student from their ideal curriculum is 0.33 in the utilitarian case, 0.28 in the mixed case, and 0.25 in the Rawlsian case. Unlike the triangular case, the welfare of the worst-off member of society is improved in the move from the pure utilitarian to the mixed case. In fact, the switch by one school to a more egalitarian charter always increases the utility of the worst-off member of society.

The fundamental reason for the difference between the triangular and the inverted triangular cases is that the former is unimodal and the latter is bimodal. A pure utilitarian system involves the two schools locating close to the center for a unimodal population distribution, but close to the extremes for a bimodal distribution. In the triangular distribution, any attempt to reduce inequality involves locating closer to the poorly-served extremes. Picking such a location shrinks the constituency of the more egalitarian school, which may lead to a greater degre of social inequality. In the inverted triangular case, any attempt to reduce inequality involves locating closer to the poorly-served center. Picking such a location tends to expand the constituency of the more egalitarian school, amplifying the overall reduction in inequality. The example illustrates that in order to understand the impact of a given institution, one must specify both the institutions' decision rules and the population's distribution of preferences.

This example uses what may appear to be the natural definition of an institution and an equilibrium. In abstract terms, an institution selects a policy taking its membership and those of the competing institutions as fixed. Individuals choose which institution to join anticipating both the division of the population between institutions and the institutional policies that will result. Equilibrium is a fixed-point in which the individuals that comprise the institution like the resulting policy at least as much as the policies of other institutions. But to cover other examples of institutional competition, such as Hotelling's model of political competition, we need to consider an alternative definition of an institution and equilibrium.

The Hotelling location model adapted to political competition concerns the platform choices of left-wing and right-wing political parties in a winner-take-all election. Hotelling shows that in equilibrium both parties choose to locate close to the center of the political spectrum (the position most favored by the median voter). This abandonment of ideology is needed to prevent the other party from winning. The general point is that the equilibrium is based on picking policies to attract members. The institution operates as 
it does not 80 much to appeal to the original members, but rather to appeal to potential members. In such cases, unlike the school example, it is inappropriate to treat memberships as fixed when the institutional choices are made. To model such situations calls for a definition of institutional equilibrium that gives an important role to the positions that other institutions adopt.

In order to develop a general model, we analyze both the membership-based approach and the position-based approach. The next section spells out these two approaches in detail and provides the corresponding definitions of equilibrium. Following the definitions, we discuss the applicability of each approach.

\section{III . A MODEL}

There are $m$ exogenously given institutions. Each institution $k$ has available a compact set of possible policy positions, $x_{k} \in X_{k} \subset R^{w}$. The vector of positions is represented by $\bar{x} \in X$, where $X$ is the product of the sets $X_{k}$. Institutions may be further constrained in their choice of $x_{k}$; the feasible set for a specific group may depend on the composition and size of its membership. We will make this constraint precise once we have defined the constituencies below.

Individual preferences vary across the population as summarized by a vector $\alpha \in R^{n}$. The utility of an $\alpha$-type joining institution $k$ given choices $\bar{x}$ is represented by a continuous utility function $U(\alpha, k, \bar{x}) .5$ The distribution of types across society is then represented by a hyperdiffuse probability measure $f$ on utility parameters $\alpha$ with compact support $A \subset R^{n}$.

Each type $\alpha$ anticipating institution positions $\bar{x}$ joins the institution $k$ that maximizes $U(\alpha, k, \bar{x})$. Since an individual is an infinitesimal part of an institution, no one person believes that they can influence the group's position by their action. We denote the membership of institution $k$ by $S_{k}(\bar{x}),{ }^{6}$

$$
S_{k}(\bar{x})=\{\alpha \in A \mid U(\alpha, k, \bar{x}) \geq U(\alpha, j, \bar{x}) \forall j\}
$$

Each institution responds to its membership through a political process. The feasible choices of an institution may be constrained by its membership. For example, an institution may be constrained to have a balanced budget. A local government of a small town would then require very high taxes to finance the production of a significant level of public goods. Another type of constraint on an institution's choice of position occurs when members are concerned with the group's size. For example, in politics, power is

s The interaction between an individual and the other members of the institution is restricted to the varieble $¥$. This is not as reatrictive as it may first appear; for example, $x_{k}$ could include the size of the group if that matters to the members.

- Typically, the set of indifferent individusls will be of measure zero and may be ignored. Otherwise, we assign individuals equally across the institutions to which they are indifferent. 
related to size. In this case, we interpret one of the components of $x_{k}$ as the institution's size; production of this good will be constrained by the actual membership. Generally, given any division of individuals between institutions, we denote the $k$ th institution's set of feasible choices by $X_{k}\left(S_{k}\right) \subset X_{k}$.

It remains to define the institutional decision-making process. Here the two alternative apprasches diverge. In the membership-besed definition, the institutional decisionmaking process of institution $k$ is defined by a policy function, $P_{k}$, which maps the memberships of all institutions into the set of feasible positions: ${ }^{7}$

$$
x_{k}=P_{k}\left(S_{1}, \ldots, S_{m}\right), \quad x_{k} \in X_{k}\left(S_{k}\right)
$$

An institutional process inputs institutional memberships, determines constraints based on these memberships, and outputs decisions.

In the position-based definition, the institutional decision-making process of institution $k$ is defined by a function, $Q_{k}$, which maps the membership of the $k$ th institution and the positions of all rival institutions into the set of feasible positions, where the feasible set for the $k$ th institution depends on the rationally anticipated membership given the fixed position of rival institutions and its own proposed position:

$$
x_{k}=Q_{k}\left(S_{k}(\bar{x}), \bar{x}_{-k}\right), \quad x_{k} \in X_{k}\left(S\left(x_{k}, \bar{x}_{-k}\right)\right) \text {. }
$$

In both definitions, the interplay between membership and position leads to a natural concern with internal consistency. Under what conditions are the institutional processes consistent with free mobility? For each of the membership-based and position-based models, we offer the natural definition of an equilibrium.

In the membership-based case, each individual rationally anticipates the membership choices of others and the resulting institutional policies, and selects the most preferred institution.

Definition 1 [MEMBERShIP-BASEd InSTITUTIONAL EqUILIBRIUM]: Given the set of institutional processes, $\left\{P_{1}, \ldots, P_{m}\right\}, \bar{x}^{*}$ is an equilibrium if and only if

$$
\bar{x}_{k}^{*}=P_{k}\left(S_{1}\left(\bar{x}^{*}\right), \ldots, S_{m}\left(\bar{x}^{*}\right)\right), \quad k=[1, \ldots, m] .
$$

In the position-based case, each individual rationally anticipates the positions that each institution will take and chooses among institutions based on these anticipated positions. Given these memberships, the institutions' predicted positions are feasible and no individual wishes to switch institution.

7 We do not consider the case of policy correspondences. 
Definition 2 [Position-BASEd InstTtutional Equilibrium]: Given the set of institutional processes, $\left\{Q_{1}, \ldots, Q_{m}\right\}, \tilde{x}^{*}$ is a pasition-based institutional equilibrium if and only if

$$
x_{k}^{*}=Q_{k}\left(S_{k}\left(\bar{x}^{*}\right), \bar{x}_{-k}^{*}\right), \quad k=[1, \ldots, m] .
$$

To appreciate the relevance of these concepts, we outline how they apply to a variety of economic and political settings. We close the section with a discussion of the out-ofequilibrium beliefs that support the membership-based and the policy-based definitions of an institution.

The membership-based definition has been applied to local public goods by Westhoff [1977] and Epple, Filimon and Romer [1984]. The policy choices are levels of taxes and transfers and the levels of supply of various public goods. Individuals take the institutional processes of the various jurisdictions as given, and determine which community to join anticipating the level of taxes and public goods that will result. Each community has a budget constraint; this is reflected in restrictions on the set of feasible tax-transfer systems within the community. The policy-based definition has also been applied in these settings; Epple and Romer [1991] consider the case of redistribution by local governments.

The membership-based definition also applies to club formation. A club is a supplier of a public good in which there are congestion externalities. There are many possible mechanisms for supplying such goods, ranging from market-based fee-paying mechanisms to quantity rationing on a first-come, first-serve basis. There are efficiency reasons for society to divide up into separate clubs. Here, the institutional process may be a written club charter that specifies how funds are to be raised and rights to use the club good allocated as a function of the membership. As with the local public good, the need to finance the facilities restricts the feasible set of policies for a club. The membership-based definition is relevant here if potential members must pay their fees before participating in the decision on fund-raising and other allocational issues. A position-based approach would apply to an entrepreneur who builds a club rationally anticipating a membership that will be self-financing.

The case of competition among political parties is more naturally phrased as positionbased, as in the Hotelling model. As institutions, a typical political party can be viewed as having a charter that calls for influencing society in certain directions. But one of the

8 An interesting case that is not covered in the current definitions, but which can be captured by a minor extension, involves competing corporate charters. Corporations have charters detailing such issues a their governance structure and how it can be changed. The manner in which corporate charters compete is in the struggle for investor funds. The primary difference between this and the other cases is that membership need not be exclusive: an individual can join several institution in degrees related to the size of the investments. It is straightforward to amend the definition to sllow both for multiple memberships, and for varying degrees of membership in each institution. 
usual requirements to have an influence is that the party attract enough members (and voters) to become a player on the local or national scene. This means that the size of the party may be an element of the $\bar{x}$ vector relevant to the decisions of an outsider on whether or not to join the party. In describing the party's set of feasible positions, its feasible supply of power depends directly on its membership. The policy chosen to attract members depends on the policies of rival parties.

The definitions of institutional equilibrium also cover standard economic cases of adverse selection, as in the insurance model of Rothschild and Stiglitz (1976). Consider two competing mutual-insurance collectives. Constrained to break even, the types of insurance contract each collective can afford to offer depends on who they attract (and who they attract determines what they choose to offer). In most models of such insurance markets, the argument is position based: each collective takes the policy of the other collective as given in determining its own policy.

The examples suggest that one of the key issues in assessing whether the membershipbased approach or the policy-based approach is applicable is whether the policy selection stage precedes or follows the final membership decisions. One possible interpretation is that the membership-based definition applies whenever the choice stage directly precedes the policy adoption stage, while the policy-based definition is designed for the more common settings in which the policy is set in order to capture members. In this vein, Epple and Romer [1991] argue that the policy-based approach is therefore the more widely-applicable and sophisticated of the two approaches.

We do not share this view. We believe that the membership-based definition is more widely applicable than the policy-based definition. The fact that policies may be designed to capture members can provide a rationale for selecting among membershipbased equilibria. There are many settings (especially those involving economic choices) in which holding to the policy-based definition may not be logically coherent. In the policybased equilibrium, each institution takes the position but not the membership of the other institutions as given. But what happens if the competitors' original positions are no longer feasible given the resulting policy choices? In such situations, it is not credible to view institutions as capable of fully committing to a strategy until the final members show up. It is therefore necessary for each institution to form a conjecture concerning the reaction of competing institutions when their actual membership gathers. In this spirit, Riley (1979) and Wilson (1980) argue that an insurance company cannot take the competitors' contracts as fixed; if a firm steals a competitor's most profitable customers, causing that firm to lose money, it is not reasonable to assume that the rival will continue to offer this contract (and this change will influence the profitability of all other contracts).

- To be fully general, one should sllow the feasible set of policies for a given group to depend not only on its own constituency, but also on the memberships of others, since the size of all institutions may infiuence the power that each individually wields. 
Whenever the fessible set of policy options is critically influenced by the actual members of the institution, it is ultimately necessary to pick a position that is feasible for the final membership. When anticipating what a rival institution will do, that decision will ultimately be a function of the rival's membership. Any equilibrium will have to be based on the true memberships that show up, as in the membership-based definition. If there are prior stages in which tentative cosilitions and institutional decisions are made, then they may help to select among such membership-based equilibria. ${ }^{10}$ This critique of position-based does not mean that we view it as irrelevant: just that it is more likely to apply in cases where the feasible set is not influenced by membership, as in competition among ideological institutions.

While these sophisticated thoughts focus around selection of equilibria in the membershipbased case, this leaves unanswered the fundamental issue of whether an equilibrium exists. The next sections explore the issue of existence. We work primarily with the membershipbased definition and then show how the results extend to the position-based definition.

\section{IV . OVERVIEW OF EXISTENCE}

In addressing the fundamental issue of existence, we will focus only on cases where equilibrium involves different institutions making distinct choices. We are not interested in trivial equilibria in which all institutions make identical choices and the population is divided as a matter of indifference in such a way that identical positions are selected. ${ }^{11}$ We provide two examples to illustrate the fact that there may be no equilibrium.

The first example is adapted from Epple and Romer [1991]. Consider individuals who differ only in their income level $y$, and who are considering which of two towns to live in. The towns must set a proportionate tax rate, $t \in[0,1]$, and also a level of government transfers, $g$. The towns are required to balance their budget. In a membership-based equilibrium, the budget would be balanced based on the current residents, while in the position-based case, the budget would be based on the rationally anticipated membership given the tax-transfer of the other town. Each individual cares only about his or her income net of taxes and transfers,

$$
U(y, g, t)=y(1-t)+g .
$$

The $(t, g)$ combination in each town is chosen by majority rule among the current members.

\footnotetext{
10 There are many different ways in which one might envisage selecting among equilibria, generally involving some mix of cooperative and noncooperative reasoning. One possible route is to say that in order to attract new people to a tentatively formed institution, it must be a Pareto improvement for the current members, a gain for the new members, and remain an equilibrium. See, for example, Prescott and Townsend (1984) and Bernheim, Peleg, and Whinston (1987).

11 However, identical choice is the relevant case in some economic contexts, such as in a pooling equilibrium in an insurance market.
} 
If there is an equilibrium in which the two towns differ, then the town that sets the higher tax rate must also set the higher level of government transfers; otherwise it will not attract any inhabitants. Without loss of generality, suppose that the first town sets the higher level of taxes and transfers. In this case, if a type $y$ is attracted to town 1, so will all those with lower income levels: the high tax-transfer town attracts the poorest inhabitants. But anyone in the poor town who has above that town's average income level will prefer to move to the rich town: they are net losers in town 1 as its richest inhabitants, and would at worst break even in town 2 as its poorest inhabitants. This implies that the poorest type of individuals must form a community by themselves, with everyone else in the second community. Even this will not be an equilibrium unless the second community chooses to set a zero tax rate. Under majority rule, whenever the median income is lower than the mean income (both with and without the poorest types), the tax rate will be positive and hence there will be no equilibrium in which the towns differ. ${ }^{12}$ This result holds whether one takes a membership-based or a position-based perspective.

A second (membership-based) example of institutional competition for which no nontrivial equilibrium exists arises in a political setting. Consider the case of two political parties choosing platforms where voters have Euclidean preferences and so join the party whose pasition is closest to their ideal point. If each party chooses the position favored by its most counter-clockwise member, the division between the two institutions continues to rotate counter-clockwise and there is never any stable point. In the figure below, the initial division of the population into institutions $S_{1}$ and $S_{2}$ leads to positions $x_{1}$ and $x_{2}$, which in turn leads to the new divisions $S_{1}^{\prime}$ and $S_{2}^{\prime}$.
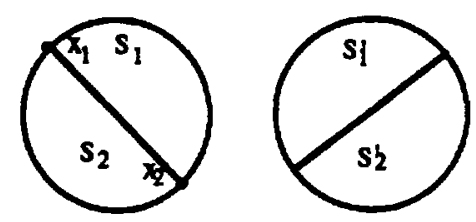

In the absence of a general existence result, we pursue three different avenues. The next section develops an existence result based on maximization of a utilitarian social welfare function. This places strong restrictions on the decision-making process, although it covers a number of interesting political applications. This is followed by a continuitybased approach. Although quite general, it relies critically on an assumption that the institutions have certain idiosyncratic features that are not inherent to the problem. To handle the cases without idiosyncrasies, we turn to the algebraic approach of Section 7 . Although existence is established, the result depends on the dimensionality of the problem. Together, these avenues help us understand the non-existence examples and provide a way forward.

\footnotetext{
12 There remains the trivial equilibrium in which both towns pick identical tax-transfer schemes and each type in the population divides evenly between the towns.
} 


\section{MAXIMIZATION APPROACH}

In this section, we open up the black box of the institution's decision-making process and focus on three specific decision rules: (1) Utilitarianism, (2) Rawlsianism, and (3) Borda Count. The institutions we consider are best viewed as political parties because we assume that the feasible set for a group, $X_{k}$, is independent of the size or composition of its membership. The political parties each compete for members through their ideological positions and voters align themselves with a party as a way of expressing their preferred position. For each of these three decision processes, we demonstrate the existence of both a membership-based and a position-based equilibrium.

A utilitarian party will choose a position so as to maximize an additive welfare function of its members' utilities.

Definition [Weighted Utilitarianism]: $P_{k}\left(S_{1}, \ldots, S_{n}\right)$ is a $\rho$-weighted utilitarian decision rule if it chooses $x_{k}$ to maximize

$$
\left[\int_{\left\{\alpha \in S_{k}\right\}} U\left(\alpha, x_{k}\right)^{\rho} f(\alpha)\right]^{\frac{1}{\rho}} .
$$

This definition may be extended to include $\rho=\infty, 0,-\infty$ through continuity arguments.

This weighted utilitarian objective function is the Hardy-Littlewood generalized mean function (also known to economists as the CES function). If $\rho=1$, the party's objective is to maximize the average utility of its members, which is the standard Benthamite version of utilitarianism. ${ }^{13}$

THEOREM 1: Consider an $m$ party competition, where each of the parties employs a common weighted utilitarian objective function to determine the outcome of its political process. Given Euclidean voter preferences $U(\alpha, x)=-\|\alpha-x\|, \alpha \in A \subset R^{n}$, a constant feasible set $X\left(S_{k}\right)=X_{k}$, and a general hyperdiffuse probability measure $f(\alpha)$ with compact support, there exists a (Pareto-optimal) membership-based equilibrium.

Proof: Consider a social planner with the power to assign voters to parties. Give this planner the objective function $L$ :

$$
L=\left[\int_{\{\alpha \in A\}} U\left(\alpha, x_{k}\right)^{\rho} f(\alpha)\right]^{\frac{1}{\rho}} .
$$

The planner chooses $\bar{x}$ and assigns the population to parties in order to maximize $L$. A solution exists since the objective function is continuous and the feasible set is compact.

\footnotetext{
13 Note that the party holds its memberahip constant in the maximization problem, so that
} maximizing the average and maximizing the sum are equivelent. 
The result of this optimization must be a membership-based equilibrium. Each person is in the party they most prefer as otherwise it would be possible to increase the sum of utilities. Additionally, each party must be maximizing the $\rho$-weighted sum of utilities of its members as otherwise the planner could increase the aggregate sum of $\rho$-weighted utilities by varying some $x_{k}$. Since this equilibrium is the result of a utilitarian social welfare maximization, the result must be Pareto optimal.

Q.E.D.

The case of weighted utilitarianism is more general than it might first appear. In particular, it includes a Rawlsian objective function as the limiting case of $\rho=-\infty$. The utilitarian summation converges to a "Leontief function" where each party evaluates a position based on the welfare of its worst-off member. ${ }^{14}$

It is pethaps more surprising that with Euclidean preferences, weighted utilitarianism also includes a political decision-making process based on a Borda count. The slight complication is that an individual must give a score to each of a potentially infinite number of alternatives. Feld and Groffman (1988) suggest the following approach. An individual of type $\alpha$ ranks position $x$ according to the measure of the set of more desirable alternatives,

$$
B(\alpha, x)=\int_{\{y: U(\alpha, y) \geq U(\alpha, x)\}}^{1 d y .}
$$

Low scores are more desirable. These scores are then aggregated across the membership,

$$
B_{i}(x)=\int_{\alpha \in S_{i}} B(\alpha, x) f(\alpha) d \alpha .
$$

Each group picks the $x$ which has the lowest aggregate Borda count summing across its members. An advantage of this Borda count rule is that a winner always exists (unlike majority rule) and there is no problem of cycles.

When voters have Euclidean preferences and the distribution of most-preferred points is hyperdiffuse, the Borda count winner is unique. It is defined by

$$
x_{i}^{*}=\arg \max \int_{\alpha \in S_{i}}-\|\alpha-x\|^{n} f(\alpha) d \alpha,
$$

where $n$ is the dimensionality of $\alpha .^{15}$ The Borda count winner is the result of a utilitarian decision rule where the Euclidean preferences are given a specific cardinalization, $U(\alpha, x)=-\|\alpha-x\|^{n}$.

Similar arguments demonstrate existence for a position-based approach to weighted utilitarianism. The result of the social planner's optimization must also be a positionbased equilibrium. If one institution was able to improve the sum of its members' utilities,

14 The value of $\rho$ provides a natural ordering of the party's concern with equality; lower values of $\rho$ imply $a$ greater weight on the utility of the less well-off members. More information on this function can be found in our paper concerning a mean voter theorem; seo Caplin and Nalebuff [19918].

13 The set of preferred alternatives to $x$ is a ball around $\alpha$ with radius $\|x-\alpha\|$. 
the sum of utilities across society would also rise and this contradicts the fact that the initial allocation was a maximum for society. This result relies heavily on the fact that individuals are concerned only with policy choices and not the size of institutions (as the choice sets are independent of the membership).

The specification of the number of parties, the dimensionality of the issue space, and the distribution of voter preferences are all quite general. The restriction to Euclidean preferences in Theorem 1 can certainly be relaxed. ${ }^{16}$ However, these existence results are limited in two important respects: all institutions must employ identical decision rules and the feasibility constraint on the positions an institution can adopt does not depend on its membership.

\section{VI . CONTINUITY APPROACH}

The continuity approach makes the assumption that an individual not only has opinions about the policy that an institution adopts, but also an idiosyncratic view of the institution. For example, there are those who prefer the Democratic party to the Republican party, even if the two parties take the same position on all observable issues. ${ }^{17}$ An individual now has a type vector $(\alpha, \epsilon)$ where the $\alpha \in R^{n}$ component refers to the explicit policies adopted, and the $\epsilon \in R^{m}$ gives the $m$-vector of institution-specific idiosyncratic factors,

$$
U(\alpha, \epsilon, i, \bar{x})=U(\alpha, i, \bar{x})+\epsilon_{i}
$$

We make certain simple continuity assumptions. We assume that the utility functions $U(\alpha, i, \bar{x})$ are continuous in $\vec{x}$ and that the joint density $f(\alpha, \epsilon)$ is hyperdiffuse and has an unbounded support.

As before, we can define the membership of each institution $k$ by $S_{k}(\bar{x})$ :

$$
S_{k}(\bar{x})=\left\{(\alpha, \epsilon) \in A \times R^{m} \mid U\left(\alpha, x_{k}\right)+\epsilon_{k} \geq U\left(\alpha, x_{j}\right)+\epsilon_{j} \forall j\right\} .
$$

The proportion of type $\alpha$ individuals who choose to join party $k$ is

$$
g_{k}(\alpha, \bar{x})=\int_{(\alpha, \epsilon) \in S_{k}(\bar{x})} f(\alpha, \epsilon) d \epsilon .
$$

Note that $f(\alpha, \epsilon)$ is a joint density, so that we need not assume $\alpha$ and $\epsilon$ are independent. ${ }^{18}$

\footnotetext{
16 The asoumption is used to guarantes that each group will make a distinct choice and that no group will end up with zero members. More generally, any utility function that ensures that $L$ is a strictly increasing function of $m$ guarantes that all parties will have distinct positions and positive membership.

17 This could arise from an incompletenes in the specification of party positions. Parties will be called upon to take positions in the future and voters must anticipate how parties will make these decisions. There is clearly room for disagreement.

18 Since probsbilities must add up to one, $\sum_{k} g_{k}(\alpha, x)=f(\alpha)=\int_{c} f(\alpha, \epsilon) d \epsilon$.
} 
Since the utility functions are continuous in $x$, it follows that $g_{k}(\alpha, \bar{x})$ will also be continuous in $\bar{x}$. Thus each institution's constituency will be a contimuous function of the positions.

The other side of the equation is to consider how institutions choose positions as a function of their constituency. Since $\epsilon$ has unbounded support, each group will always have a positive mass. Thus we need not be concerned with the outcome of a decision rule for a group with no members. The institution's decision process chooses an outcome from $X_{k}\left(S_{k}\right)$ based on the preferences of its members. We assume that the decision is continuous in the weak topology. ${ }^{19}$ This would be satisfied, for example, if the decision rule is the expectation of any continuous function.

With these assumptions, one can prove existence of an equilibrium by a standard application of Brouwer's fixed point theorem. Given any vector of policies $\bar{x}$, we identify the corresponding constituencies, $\left\{g_{1}(\alpha, \bar{x}), \ldots, g_{m}(\alpha, \bar{x})\right\}$. We then input this set of constituencies into the policy functions to produce a new set of positions $\bar{x}^{\prime}$. By assumption, the domain is compact, and both the mapping from $\bar{x}$ into constituencies and the mapping from constituencies to policies are continuous. Brouwer's theorem then ensures that a fixed point exists, and it is immediate that such a fixed point constitutes an equilibrium.

The existence argument for a position-based equilibrium is essentially identical. As in the membership-based case, continuity of the utility functions in $x$ implies that each institution's membership is a continuous function of the positions. The assumption that the institution's decision rule, $x_{k}=Q_{k}\left(S_{k}(\bar{x}), \bar{x}_{-k}\right)$, is continuous in the weak topology allows application of Brouwer's theorem to establish existence. While the continuity of the decision rule is the same assumption, the meaning is now different since the constraint sets are allowed to vary with the choice of $x_{k}$.

There are two reasons to consider this type of an idiosyncratic choice model. In certain settings, this may be an accurate description of how individuals make decisions. A second reason is to use the idiosyncratic element as a mathematical device to approximate the outcome with no noise. We can consider a convergent subsequence of fixed points and examine the limit as the variance of $\epsilon$ approaches zero. If the parties choose distinct positions in the limiting equilibrium, this solution is also an equilibrium for the problem with no noise. ${ }^{20}$ But there is the possibility that as the noise approaches zero, two institutions' positions will approach each other. It is precisely the case of equal choice that creates an existence problem when there is no idiosyncratic element. If two or more groups pick

\footnotetext{
19 The intitutions' positions are continuous in the conditional probability distribution of its memberahip and in the mase of its membership.

20 Although we must be careful that each institution has positive membership, $S_{i} \neq 0$. Otherwise, the outcome of the decision-making proceas is indeterminate.
} 
identical positions, there is no way to divide the population while preserving continuity of the memberships. ${ }^{21}$ As a result, we must consider an entirely different approsch.

\section{VII . AN INDEX THEORY APPROACH}

In this section, we consider a general political process for two-party competition while imposing restrictions on the type of voter preferences. The novel results of this paper are Theorems 2 and 3, which prove existence of an equilibrium for competition with an odd number of dimensions.

We restrict individual preferences so that each individual evaluates an institution by a weighted sum of its perceived benefits. The population differs only in the weights used to evaluate benefits. The utility benefits are determined by a continuous function $t$ which maps the $w$-dimensions of the position into an $(n+1)$-dimensional vector of utility benefits. For example, the value of national defense and the evaluation of a budget deficit may be complicated functions of the position on taxes and trade barriers. In our framework, members have a common assessment of the benefits from each position, but differ over how they value these benefits.

Assumption A1 (Linear Preferences): Preferences can be represented in a linear form:

$$
U(\alpha, x)=\sum_{k=1}^{n} \alpha_{k} t_{k}(x)+t_{n+1}(x)
$$

where $U: R^{n} \times X \mapsto R$, and the functions $t_{k}(x)$ continuously map the institution's position into a utility valuation. Furthermore, distinct positions have distinct benefits: given $x_{i} \neq x_{j}, \exists \alpha$ s.t. $U\left(\alpha, x_{i}\right) \neq U\left(\alpha, x_{j}\right)$

Assumption A1 is based on Grandmont's [1978] development of intermediate preferences. The restriction implies a separability of issues in determining individual preferences. For ease of notation, we define $t(x)=\left(t_{1}(x), \ldots, t_{n}(x)\right)$ and then represent utility as

$$
U(\alpha, x)=\alpha \cdot t(x)+t_{n+1}(x) .
$$

The dimensionality of individuals' preferences is determined by the dimensionality of the $\alpha$ vector. But the components of $\alpha$ are relevant only to the extent that there is any variation in the population. Thus, we are really interested in the number of dimensions in which the $\alpha$ differ. The point of A2 is to ensure that there is a zero mass of individuals in any hyperplane and that any two individuals have distinct preferences. In particular, this implies that given two distinct institution positions, the set of indifferent individuals is of measure zero. For convenience, we normalize the total population to unity.

\footnotetext{
21 In fact, whenever there is the non-exiatence of an equilibrium without probabiliatic choice it must be the case that the party positions approach each other in the probabilistic choice model as the noise goes to zero.
} 
Assumption A2 (Hyperdiffuse Density, Distinct Preferences): The probability density of individuals' utility parameters is hyperdiffuse over its compact support, $A$. Within this support, preferences are distinct; for any two elements $\alpha$ and $\alpha^{\prime} \in A$, there exists some pair $\left(x_{i}, x_{j}\right) \in X$ such that type $\alpha$ strictly prefers $x_{i}$ to $x_{j}$ and type $\alpha^{\prime}$ strictly prefers $x_{j}$ to $x_{i}$.

The linear preference model covers many of the standard utility functions used in economics and we give two examples below.

- Euclidean Preferences: Each person joins the group whose position is closest to his or her most-preferred point, $\alpha: U(\alpha, x)=-\|x-\alpha\|^{22}$

- Linear Preferences: Each person evaluates a position by a weighted sum of the elements, $U(\alpha, x)=\alpha \cdot x$. With slight modifications, this covers the CES, Cobb-Douglas, logit, and probit models (see Caplin and Nalebuff, 1991b).

In the case of linear preferences, we must be careful about the choice of the support $A$. Since any monotonic transformation of the utility function leads to the same preferences, a person of type $\alpha$ and one of type $2 \alpha$ have identical preferences. Thus it is appropriate to scale the $\alpha$ vector so that it always lies on the unit sphere, $A=S^{n-1}$. If all the elements of $\alpha$ are positive, it is appropriate to scale the $\alpha$ vector so that it always lies on the unit simplex, $A=\Delta^{n-1}$. $^{23}$

The value of the linear utility functions is that we only need consider divisions of the population defined by a hyperplane. For any two distinct positions $x_{1}, x_{2}$, there exists a hyperplane with the members of institution 1 on one side and those of institution 2 on the other side. The set of people who choose to join institution 1 over 2 is defined by

$$
S_{1}(\bar{x})=\left\{\alpha \in A \mid \alpha \cdot\left[t\left(x_{1}\right)-t\left(x_{2}\right)\right] \geq t_{n+1}\left(x_{1}\right)-t_{n+1}\left(x_{2}\right)\right\}
$$

This hyperplane has a gradient vector $\pi$, which we normalize to unit length, and intercept $b$, also normalized:

$$
\pi=\frac{t\left(x_{1}\right)-t\left(x_{2}\right)}{\left\|t\left(x_{1}\right)-t\left(x_{2}\right)\right\|}
$$

22 These preferences are equivalent to $U(\alpha, x, x)=-[x \cdot x-2 \alpha \cdot x+\alpha \cdot \alpha]$, which is in the linear form once the irrelevant term $(\alpha \cdot \alpha)$ is removed.

23 All the as would be poaitive, for example, if every agrees that a lower budget deficit is a desirable platform but people differ on the intenaity of this preference. This rules out the Euclidean model in which individuals choose the position closest to their most-preferred point since there in no longer unanimity about the desirability of movement in any particular direction. The benefita need not be "goods" as opposed to "bads". For example, one of the as may be the person's income and the corresponding $t$ could be a tax rate. To convert the bad into a good, we transform the tax rate into an after tax income rate, so that individuals evaluate $\alpha(1-t)$ and the position in viewed as a benefit rather than a cost. The general point is that for each of the $n$-dimensions of "benefits," all individuals lie in a half-epace and by a reorientation of the benefit we can position this half-space in the positive orthant without any lose of generality. 


$$
b=\frac{t_{n+1}\left(x_{2}\right)-t_{n+1}\left(x_{1}\right)}{\left\|t\left(x_{1}\right)-t\left(x_{2}\right)\right\|}
$$

The orientation of the hyperplane tells us which people go to which group. In our notation, the gradient vector points into the constituency of group 1. Thus we can characterize $S_{1}$ and $S_{2}$ by $(\pi, b)$. This allows us to express the institutions' decision rules as functions of the dividing hyperplane, $x_{i}=P_{i}(\pi, b)$.

To prove existence of equilibrium, we need some additional assumptions concerning the policy functions $P_{i}(\pi, b)$. The best way to motivate these assumptions is to outline the structure of the proof of the existence result, Theorem 2.

The first stage of the proof involves the definition of a continuous mapping from the space of hyperplanes into itself. Note that each hyperplane $(\pi, b)$ defines the membership of the two groups. Given non-empty memberships, the decision processes lead to a pair of positions $P_{1}(\pi, b)$ and $P_{2}(\pi, b)$. Provided these choices are distinct, they in turn imply a division of the population back into two groups characterized by a new dividing hyperplane. It is this mapping that forms the basis of our existence proof. In order to define the mapping, we require that institutions with memberships on opposite sides of a hyperplane make distinct choices.

Assumption A3 (Distinct Choices): Two institutions whose memberships lie on opposite sides of a hyperplane will choose distinct positions: $P_{1}(\pi, b) \neq P_{2}(\pi, b)$.

Two institutions whose memberships lie in opposite half-spaces have quite distinct constituencies. Thus it is not unreasonable to assume they will never choose the same platform. Note that this motivation for A3 uses the assumption that different $\alpha$-types have distinct preferences (A2). Otherwise, we might be able to divide the population into two half spaces and yet find identical preferences in both groups.

As would be expected, one necessary condition for establishing existence of an equilibrium via a fixed point argument is continuity of the decision-making process.

Assumption A4 (ContinutTy): $P_{i}(\pi, b)$ is continuous in $(\pi, b)$.

This condition may also look innocuous but it has some subtle implications. In particular, the choice of $x_{k}$ depends both on the preferences of the group and its constraint set. Thus our continuity assumption has implications for how $X\left(S_{k}\right)$ varies with $(\pi, b) .^{24}$

We make an additional assumption about preferences and the decision rules in order to ensure that in any equilibrium both institutions will have strictly positive mass. One way to ensure this is to assume that groups which are sufficiently small tend to expand.

\footnotetext{
24 For example, if having a strict majority is of primary importance in an election, this could induce a discontinuity in a political party's feasible set.
} 
This would be true if small groups could design a policy that was tailor fitted to their constituency and the members preferred this custom position to the more anonymous options from a larger group. A second route is to assume that small groups lose some members. Following the lines suggested by Westhoff [1977], a town that is too small may not be viable. ${ }^{25}$

Assumption A5 (VIABILITY OF Small InSTITUTIONS): There exists a size $1 / 2>$ $\mu>0$ such that if one institution has population less than or equal to $\mu$, then given the position chosen by it and its competitor, at least some type of individual located in the larger group will prefer affiliating with the smaller group.

Assumption A5' (CONTRACTION OF SMALl InSTITUTIONS): There exists a size $1 / 2>\mu>0$ such that if one institution has population less than or equal to $\mu$, then given the position chosen by it and its competitor, at least some type of individual located in the smaller group will prefer affliating with the larger group.

A5 and $A 5^{\prime}$ are joint statements about the decision-making process and the feasibility constraints. We could reduce either to a statement solely about decision-making process by requiring the result to hold for any feasible choice made by the two institutions.

With Assumptions A1-A5, we are able to apply index theory to construct a continuous mapping from the space of hyperplanes to itself that has a fixed point, for cases in which the dimensionality of the preference space is odd. Unfortunately, the index theorem does not allow us to discriminate between situations of equilibrium, in which we map back to the same division, and situations where the orientation of the hyperplane is reversed, so that all individuals switch institutions. To rule out such reversals, it is enough to assume that equally-sized institutions never reverse positions. Starting from a position of equal size, someone in each group must prefer their own institution's position to that of the rival. Note that this is a very weak form of positive responsiveness of institutions to their membership. ${ }^{20}$

25 The tax example from Section 3 shows that without one of these assumptions, non-existence can occur. In that example, a small rich town expands, while a small poor town contracts, so that neither $\mathrm{A} 5$ nor $A 5^{\prime}$ are valid.

26 Epple and Romer (1991) conatruct an example in which all members of one institution prefer the policy selected by the other, and vice versa, contradicting the spirit of A6. To see how this can arise, consider a population differentiated by income in which a large poor community sets a lump sum tax to finance public good supply, and a small rich community finances the public good with a proportionate tax. Suppose they end up supplying precisely the same amount of the public good. It is then atraightforward to conotruct an exemple in which all the richer individuals would pay less under the lump sum tax scheme, while all the poorer individualo would pay leas under the proportionate tax acheme. In this case, everyone would like to switch communities. 
assumption a6 (Weak Pareto Condition): Two equal-sized institutions never choose positions in such a way that all members of one group are attracted to the other group's position: $\int_{S_{1}} f(\alpha) d \alpha=\int_{S_{2}} f(\alpha) d \alpha \Rightarrow$

$$
\begin{gathered}
U\left(\alpha, P_{1}(\pi, b)\right)>U\left(\alpha, P_{2}(\pi, b)\right) \text { for some } \alpha \in S_{1} \\
\text { and } U\left(\alpha, P_{2}(\pi, b)\right)>U\left(\alpha, P_{1}(\pi, b)\right) \text { for some } \alpha \in S_{2} .
\end{gathered}
$$

With these assumptions, we are able to establish existence of a membership-based equilibrium when the preference space has an odd number of dimensions.

THEOREM 2: Under Assumptions A1-A6, there exists a membership-based equilibrium for $\alpha \in R^{n}, n$ odd. In this equilibrium, both institutions will have strictly positive mass.

ProOf: Appendix A.

One curious aspect of this theorem is the role of the dimensionality. To see the mathematical reason for this, it is convenient to first restrict attention to the cases with $t_{n+1}(x) \equiv 0$ so that $b=0$ and all hyperplanes pass through the origin. Thus our continuous function $\pi \mapsto \pi$ maps from the unit sphere to itself. ${ }^{27}$ When does it follow that such a mapping has a fixed point? Unfortunately, there is no general result establishing the existence of a fixed point.

As we have seen, an example of a political decision rule for which no equilibrium exists is the rotation process. Each party chooses the position most favored by the most counterclockwise member of it party. As a result, the division between the two parties continues to rotate counter-clockwise and there is never any stable point. One way to summarize the problem is to go to the surface of the unit sphere and place the orthogonal vector that points in the "counter-clockwise" direction. Note that this vector changes continuously along the surface and yet has no zeros. This negative result seems at first to present a complete roadblock and suggest the need for a new line of reasoning. Remarkably, this counter-example is a problem only in even numbered dimensions..$^{23}$

This positive result for odd dimensions is sometimes referred to known as the "hairy ball" theorem, and is a corollary to the more general Lefschetz fixed-point theorem. The

\footnotetext{
27 In this example, we will assume that the support of $\alpha$ contains a ball around the origin.

28 The rotation mapping deecribed earlier is not continuous in the dividing line. The problem occurs when one party contains the entire population. In that case, there is no moet counterclockwise point. This problem can be fixed by taking the most counter-clockwise point until a party has $90 \%$ of the total mase. Thereafter, we take a weighted average of the center of gravity of the set and the most clockwise point, where the woight on the center of gravity goes to one as the party mase approaches one. There may be other potential barriers to existence; woe Rose-Ackerman [1979] and Stiglitz [1977].
} 
hairy ball theorem states that any continuous vector field on the surface of an odddimensional sphere must have a zero. For example, since the winds on the surface of the (three-dimensional) earth change direction continuously, somewhere on the earth's surface there is no wind. Or, more colloquially, if one continuously combs the hairs on the surface of a odd-dimensional sphere, then somewhere there must exist a cowlick.

Before dismissing the odd-even result as a curious mathematical artifact, it may help to understand why one cannot simply "fix up" the problem for the even-numbered dimensions. In particular, it might seem that we could just add another "fake" dimension (a dimension that no individual cares about), solve the problem in one higher dimension, and use that fixed point. While any two-dimensional problem can always be embedded in a three-dimensional setting, it cannot be done while maintaining our continuity assumptions. Consider what happens when two institutions differ only in the "fake" dimension. At that point, all individuals are indifferent between the two institutions. How should they be divided up? While it is possible to split the density in half and give each group identical memberships, note that with the slightest change in any of the true dimensions, the resulting composition of the memberships will change in a discontinuous manner. Consequently, the institutions' positions will change discontinuously and thus the fixedpoint theorem does not apply.

We now briefly indicate how to demonstrate a similar existence theorem for the position-based model. For simplicity, we replace the $t(x), t_{n+1}(x)$ with $x$. Thus preferences are $U(\alpha, x)=\alpha \cdot x .^{20}$ To demonstrate existence, we maintain A2, strengthen A3 to include undominated choices, and replace assumptions A4-A5 with their direct analogs. Again for simplicity, we assume that all the elements of $\alpha$ are positive, $\alpha \in \Delta^{n-1}$.

Assumption B3 (Distinct and Undominated Choices): In two-institution competition, groups whose constituents lie on opposite sides of a hyperplane will choose distinct and undominated positions: $\min _{k}\left\|Q_{1}\left(S_{1}\left(x_{1}, x_{2}\right), x_{2}\right)-Q_{2}\left(S_{2}\left(x_{1}, x_{2}\right), x_{1}\right)-k(1,1, \ldots 1)\right\|>$ $0 . .^{30}$ Since all individuals lie in $\Delta^{n-1}$, if the two positions differ by a unit vector, then all individuals will prefer one position to the other.

This assumption that institutions do choose undominated positions need only hold for group of some minimum size, as defined below in B5.

Assumption B4 (CONTINUITY): $Q_{i}\left(S_{i}(\bar{x}), \bar{x}_{-i}\right)$ is continuous in $\left(S_{i}, \bar{x}\right)$.

\footnotetext{
29 The reason for this change is that otherwise we would have to constantly refer to the choice of $t(x)$ rather than $x$. It is simpler to relabel the choice of $x$ as that of a $t(x)$ and then adjust the feasibility constraints accordingly.

30 This aseumption extends to include points on the boundary. For any given division $S_{1}^{*}, S_{2}^{*}$, consider all the points $\left(x_{1}, x_{2}\right)$ that lead to this division. We assume that oven for the limit points $x_{1}=x_{2}=x, Q_{1}\left(S_{1}^{*}, x\right)-Q_{2}\left(S_{2}^{*}, x\right) \neq k(1,1, \ldots 1)$.
} 
AsSUMPTION B5 (VIABILITY OF SMALl InSTITUTIONS): There exists a size $1 / 2>$ $\mu>0$ such that if one institution has population less than or equal to $\mu$, then given the position chosen by it and its competitor, at least some type of individual located in the larger group will prefer affiliating with the smaller group.

Assumption B5' (Contraction OF SMALl Institutions): There exists a size $1 / 2>\mu>0$ such that if one institution has population less than or equal to $\mu$, then given the pasition chosen by it and its competitor, at least some type of individual located in the smaller group will prefer affiliating with the larger group.

The final two assumptions are more distinct from the membership-based counterparts. First, we replace the assumption that there are no reversals (A6) between equal-sized institutions with the assumption that equal-sized institutions do not choose to imitate the position taken by the rival institution. Finally, we add a new assumption that is designed to bound the space of possible institutional policies.

AsSUMPTION B6 (No ImITATION OF EqUALS): Given two equal-sized institutions, neither adopts the pasition of its rival:

$$
\int_{S_{1}} f(\alpha) d \alpha=\int_{S_{2}} f(\alpha) d \alpha \Rightarrow Q_{i}\left(S_{i}\left(x_{1}, x_{2}\right), x_{j}\right) \neq x_{j}
$$

Assumption B7 (BoundEDness): There exists $R \leq \infty$ such that

$$
\left\|x_{j}\right\| \leq(2 \sqrt{n}+1) R \Rightarrow\left\|Q_{i}\left(S_{i}\left(x_{i}, x_{j}\right), x_{j}\right)\right\| \leq R, \quad(i, j)=\{(1,2),(2,1)\} .
$$

We assume that each set $X_{i}$ contains this $(2 \sqrt{n}+1) R$ ball.

With these assumptions, we again use the Lefschetz fixed-point theorem to establish existence of an equilibrium when the preference space has an even number of dimensions.

Theorem 3: For $U(\alpha, x)=\alpha \cdot x, A 2$, and B3-B7, there exists a position-based equilibrium for $\alpha \in \Delta^{n-1}, n$ even. In this equilibrium, both institutions will have strictly positive mass.

ProOf: Appendix A.

\section{VIII . CONCLUSIONS}

This research is motivated by our desire to integrate institutions more fully into economic theory. Typically, researchers focus either on the influence of the economic environment on the structure of institutions, or on the influence of institutions on economic outcomes. We have explored a model of institutional competition that allows us to focus 
on the essential interactions between the economic environment and the structure of institutions: the policy that each institution adopts depends on the memberships, and the membershipe depend upon the policies of all institutions.

The theory has potential applications to such diverse topics as: local public finance; the platforms of political parties; the relationship between shareholders and corporate policy; the structure of political coslitions such as the EEC; and self-selection into contracts. Although there is no general existence result, this paper provides three of the basic elements to carry out these applications. First, we offer appropriately abstract definitions of an institution. Second, we specify the corresponding equilibrium concepts. Third, we show how a maximization approach, a continuity approach, and an index theory approach provide existence results for multidimensional competition and institutional decision processes other than majority rule. 


\section{IX . BIBLIOGRAPHY}

AUsten-SmTt, D. AND J. Banks (1991b): "Elections, Coalitions, and Legislative Outcomes," American Political Science Review 82, 405-422.

BARON, D. AND J. FEREJOHN (1987): "Bargaining in Legislatures," American Economic Review 77, 303-309.

Baron, D. (1991a): "Government Formation and Endogenous Parties, " Stanford University Working Paper.

Baron, D. (1991b): "A Spatial Bargaining Theory of Government Formation in a Parliamentary System," American Political Science Review 85, 137-164.

Bernheim, B., Peleg, B. AND M. Whinston (1987): "Coalition-Proof Nash Equilibria 1. Concepts," Journal of Economic Theory 42, 1-12.

BLACK, D. (1948): "On the Rationale of Group Decision-Making," Journal of Political Economy 56, 23-34.

Buchanan J. (1965): "An Economic Theory of Clubs," Economica 32, 1-14.

Caplin, A. And Nalebuff, B. (1991a): "Aggregation and Social Choice: A Mean Voter Theorem," Econometrica 59, 1-24.

Caplin, A. ANd Nalebuff, B. (1991b): "Aggregation and Imperfect Competition: On the Existence of Equilibrium," Econometrica 59, 25-60.

Casella, A. And J. Feinstein (1990): "Public Goods in Trade: On the Formation of Markets and Political Jurisdictions, "Stanford University Working Paper.

EpPle, D., R. Filimon, ANd T. ROMER (1984): "Equilibrium Among Local Jurisdictions: Toward an Integrated Treatment of Voting and Residential Choice," Journal of Public Economics 24, 281-308.

EPPLE, D. AND T. ROMER (1990): "Mobility and Redistribution," Journal of Political Economy 99, 828-57.

Grandmont, J.M. (1978): "Intermediate Preferences and the Majority Rule," Econometrica 46, 317-330.

Greenberg, J. (1983): "Local Public Goods with Monopoly: Existence and Optimality of a General Equilibrium," Journal of Economic Theory 30, 17-33.

Greenberg, J. AND S. Weber (1986): "Strong Tiebout Equilibrium under Restricted Preferences Domain," Journal of Economic Theory 38, 101-117.

Feld, S. ANd B. Groffman (1988): "The Borda Count in n-dimensional issue space," Public Choice 59, 167-76.

Munkres J. (1984): Elements of Algebraic Topology. Menlo Park, CA: Addison-Wesley.

Palfrey, T. and H. Rosenthal (1985): "Voter Participation and Strategic Uncertainty," American Political Science Review 76, 62-78.

Prescott, E. AND R. Townsend (1984): "Pareto Optims and Competitive Equilibria with Adverse Selection and Moral Hazard," Econometrica 52, 21-45. 
Richter, D. K. (1982): "Weakly Democratic Regular Tax Equilibria in a Local Public Goods Economy with Perfect Consumer Mobility," Joumal of Economic Theory 27, $137-162$.

RILEY, J. (1979): "Informational Equilibrium," Econometrica 47, 331-59.

ROSE-ACKERMAN, S. (1979): "Market Models of Local Government: Exit, Voting, and the Land Market," Journal of Urban Economics 6, 319-337.

RothschILD, M. AND J. STIGLITZ (1976): 'Equilibrium in Competitive Insurance Markets: An Essay on the Economics of Imperfect Competition," Quarterly Journal of Economics 90, 629-50.

ScOTChMER, S. (1983): "Profit-Maximizing Clubs: Two-Stage Symmetric Nash Equilibrium, " Harvard University Working Paper, HIER 1030.

STiglitz, J. (1977): "The Theory of Local Public Goods," in The Economics of Public Services, ed. by M. Feldstein and R. Inman. London: Macmillan, 274-333.

Stigler, G. (1957): "The Tenable Range of Functions of Local Government, " In Federal Expenditure Policy for Economic Growth and Stability, by the Joint Economic Committee. Washington: Government Printing Office..

Trebout, C. (1956): "A Pure Theory of Local Expenditures," Journal of Political Economy $64,416-424$.

Westhoff, F. (1977): "Existence of Equilibria with a Local Public Good," Journal of Economic Theory 14, 84-112.

Westhoff, F. (1979): "Policy Inferences from Community Choice Models: A Caution," Journal of Urban Economics 6, 535-549.

WiLsON, C. (1980): "The Nature of Equilibrium in Markets with Adverse Selection," Bell Journal of Economics 11, 108-30. 


\section{$\mathrm{X}$. APPENDIX}

This Appendix contains the proofs of Theorems 2 and 3 concerning existence of membership-based and position-based equilibrium in the model of Section 7. Both proofs come in four parts. The first part of each proof defines the domain of a mapping. The second part provides a detailed construction of a continuous mapping from this domain into itself. The third part involves application of index theory to prove existence of a variety of fixed points. The final part proves that at least one of the fixed points is an equilibrium. The index theorems used in the third part of each proof follow as Theorem 4; this theorem was prepared with the assistance of James Munkres.

Theorem 2: Under Assumptions A1-A6, there exists a membership-based equilibrium for $\alpha \in R^{n}, n$ odd. In this equilibrium, both institutions will have strictly positive mass.

ProOf: We present the proof in the four stages referred to above.

A. Defining the domain: $D_{\mu}$ is the compact set of all $(\pi, b)$ vectors in $S^{n-1} \times R$ such that the population on each side of the hyperplane is at least $\mu$, where $\mu$ is the value from A5 that guarantees the viability of small institutions. Since the support of $\alpha$ is compact, for each $\pi$ there exists $b_{\min }(\pi)$ and $b_{\max }(\pi), b_{\min }(\pi)<b_{\max }(\pi)$, such that the population division determined by $\left(\pi, b_{\min }(\pi)\right)=(\mu, 1-\mu)$ and the population division determined by $\left(\pi, b_{\max }(\pi)\right)=(1-\mu, \mu)$. Both $b_{\min }(\pi)$ and $b_{\max }(\pi)$ vary continuously with $\pi$. Since $(\pi, b)$ and $(-\pi,-b)$ define the same hyperplane, but with opposite orientation, if $(\pi, b)$ is in the domain, so is $(-\pi,-b) .^{1}$

B. Construction of the mapping: Given $(\pi, b)$, the memberships of the two institutions are well-defined. The members choose positions $x_{1}=P_{1}(\pi, b), x_{2}=P_{2}(\pi, b)$. With A3, the distinct institutions choose distinct positions, so that $x_{1} \neq x_{2}$, and the result of free mobility is a new division of the population into groups defined by a hyperplane $\left(\pi^{\prime}, b^{\prime}\right)$. However, our mapping is not from $(\pi, b)$ to $\left(\pi^{\prime}, b^{\prime}\right)$. We use $\pi^{\prime}$ with a different intercept $b^{\prime \prime}$, which depends on the value of a fixed scale parameter, $\lambda \in(0,1)$, in the following manner. ${ }^{2}$

To determine the new intercept value, define $M_{i}$ as the original population of party $i$ under $(\pi, b)$, and $M_{i}^{\prime}$ as the population of party $i$ according to $\left(\pi^{\prime}, b^{\prime}\right)$. The definition of the final intercept $b^{\prime \prime}$ divides into cases depending on the value of $M_{1}, M_{1}^{\prime}$ and $\lambda$ as follows:

1 Note that $b_{\min }(x)=-b_{\max }(-\pi)$.

2 While $\lambda$ is presently fixed, in part $D$ of the proof we will consider a sequence of mappings in which $\lambda$ approaches zero. 
Case 1 (Interior): If $\mu \leq M_{1}+\lambda\left(M_{1}^{\prime}-M_{1}\right) \leq 1-\mu$, then we define $b^{\prime \prime}$ by taking the $\pi^{\prime}$ hyperplane and shifting it until the population in party 1 is exactly $M_{1}+\lambda\left(M_{1}^{\prime}-M_{1}\right)$.

Case 2 (Boundary): If $M_{1}+\lambda\left(M_{1}^{\prime}-M_{1}\right) \leq \mu$, then we set $b^{\prime \prime}=b_{\max }\left(\pi^{\prime}\right)$, while if $M_{1}+\lambda\left(M_{1}^{\prime}-M_{1}\right) \geq 1-\mu$, then we set $b^{\prime \prime}=b_{\min }\left(\pi^{\prime}\right)$.

In words, this means that we map from the original $\pi$ to the new $\pi^{\prime}$, but set the intercept so that the population is a weighted average of the original population and the new population. ${ }^{3}$ In Case 1 above, the weight $\lambda$ on the new population results in a mapping back into $D_{\mu}$ itself, and this then defines the final mapping. In Case 2, the use of these fixed weights would result in a mapping outside the domain: the hyperplane is then shifted back to the boundary of the domain.

With this, we have constructed a mapping from $D_{\mu}$ to itself. The fact that the mapping is continuous follows from Assumption A4. With A4, small changes in $(\pi, b)$ induce correspondingly small changes in the policies $P_{1}(\pi, b)$ and $P_{2}(\pi, b)$, which in turn produce continuous changes in the division of the population between the institutions. Hence the mapping from $(\pi, b)$ to $\left(\pi^{\prime}, b^{\prime}\right)$ is continuous, as is the mapping from $b^{\prime}$ to $b^{\prime \prime}$ defined above, confirming overall continuity of the mapping.

C. Application of Index Theorem. We have chosen this mapping in order to apply the Lefschetz fixed-point theorem. The conclusion of the theorem, as stated in Theorem 4 below, is that there exists $(\pi, b) \in D_{\mu}$ such that $(\pi, b)=\left(\pi^{\prime}, b^{\prime \prime}\right)$ or $(\pi, b)=\left(-\pi^{\prime},-b^{\prime \prime}\right)$ : there exists some hyperplane that maps back to itself, but its orientation might be reversed. We refer to all such hyperplanes as fixed points of the mapping.

D. Existence of Equilibrium. There are four qualitatively distinct types of fixed points, depending on whether the orientation is preserved or reversed, and whether we are in Case 1 (the interior case) or Case 2 (the boundary case) in the definition of the mapping.

(1) An orientation-preserving interior fixed point. In this case, the original population and final populations are the same, as is the gradient vector, and hence we have an equilibrium.

(2) An orientation-preserving boundary fixed point. This is impossible, since it involves an institution with original population $\mu$ shrinking to a final population below $\mu$ while the dividing hyperplane maintains the same normal. Thus the smaller institution shrinks to a subset of its original membership. This contradicts A5 which states that someone new is attracted to a small institution with population $\mu$.

3 This mapping is motivated by the construction introduced in Westhof [1977]. 
(3) An orientation-reversing interior fixed point. For this to happen, it is necessary that an initial population division $\left(M_{1}, M_{2}\right)$ leads to a reversed population division $\left(M_{2}, M_{1}\right)$. This cannot be immediately ruled out.

(4) An orientation-reversing boundary fixed point. This requires that an original population of size $\mu$ results in a population of $1-\mu$ in the final mapping. This is impossible because even the maximum possible shift is not big enough to bring a population of $\mu$ up to $1-\mu$.

To prove that there is an equilibrium, we consider a sequence of mappings replacing $\lambda$ by $\lambda_{n}=\lambda / n$. By the above argument, each such map has an interior fixed point. To prove existence of an equilibrium, we need only show that not all of these fixed points can be orientation-reversing. If all fixed points were of this type, then we could construct a sequence, $\left(\pi_{n}, b_{n}\right)$ converging to $(\hat{\pi}, \hat{b})$ in which each $\left(\pi_{n}, b_{n}\right)$ maps to $\left(-\pi_{n},-b_{n}\right)$. Note that $(\hat{\pi}, \hat{b})$ must divide the population into two equal portions, $M_{1}=M_{2}=1 / 2$, since any reversal of population sizes must become infinitesimal as $\lambda_{n}$ approaches zero. In addition we know by continuity that $(\hat{\pi}, \hat{b})$ maps back to the gradient vector $-\hat{\pi}$. Since the orientation of the hyperplane has been reversed, at least one of the new institutions contains a superset of the other institution's original membership. Starting with equal populations, one group has taken all of the other's constituents, violating A6 and establishing the theorem.

Q.E.D.

Theorem 2 applies when the domain of $\alpha$ is $R^{n}$, with $n$ odd. The case of $\alpha \in S^{n-1}$, with $n$ odd follows directly. The case of $\alpha \in \Delta^{n-1}$ is similar to a problem in one lower dimension, without the constraint to the simplex and hence equilibrium exists for $n$ even; this is the case we consider in Theorem 3 below.

To change the proof to cover the case of nonvisble small institutions [A5'] is straightforward. We simply shift the direction of the mapping, replacing $\lambda$ by $-\lambda$. This is the approach used by Westhoff. In this variation, an institution that loses members is rewarded with a greater population. This points to an instability of the equilibrium [Westhoff [1979]. We can also use the above proof structure to extend the one-dimensional $m$ institution existence proof of Westhoff beyond the case of majority rule to a broader class of political processes.

Theorem 3: For $U(\alpha, x)=\alpha \cdot x, A 2$, and B3-B7, there exists a position-based equilibrium for $\alpha \in \Delta^{n-1}, n$ even. In this equilibrium, both institutions will have strictly pasitive mass.

ProOf: We present the proof in the four stages referred to above.

A. Defining the domain: We define the domain $D_{\mu}$ of the mapping in two stages. At the first stage, we consider all $\left(x_{1}, x_{2}\right)$ vectors with $\left\|x_{i}\right\| \leq(2 \sqrt{n}+1) R$ and $\min _{k} \| x_{1}-x_{2}-$ 
$k(1,1, \ldots 1) \|>0$, such that the population in each of $S_{1}\left(x_{1}, x_{2}\right), S_{2}\left(x_{1}, x_{2}\right)$ is at least $\mu$ (here $\mu$ is the value from B5 that guarantees the visbility of small institutions and $R$ is the value from B7). To arrive at the second-stage definition of the domain, we pick any pair $\left(x_{1}, x_{2}\right)$ that satisfies the first-stage definition, and compute the corresponding policy choices, $Q_{1}\left(S_{1}\left(x_{1}, x_{2}\right), x_{2}\right)$ and $Q_{2}\left(S_{2}\left(x_{1}, x_{2}\right), x_{1}\right)$.

With Assumption B3, we know that $Q_{1}\left(S_{1}\left(x_{1}, x_{2}\right), x_{2}\right)$ and $Q_{2}\left(S_{2}\left(x_{1}, x_{2}\right), x_{1}\right)$ differ. In fact, we know there is a minimum distance $d_{\min }>0$, between $\left(Q_{1}-Q_{2}\right)$ and the 45degree line. To see this, pick any fixed hyperplane $(\pi, b)$ that has at least $\mu$ population on both sides, and consider the set of all $\left(x_{1}, x_{2}\right)$ pairs that give rise to this division. With B3 it is not possible to find a convergent sequence of such pairs $\left(x_{1}(n), x_{2}(n)\right)$ such that for some $k,\left\|Q_{1}\left(S_{1}\left(x_{1}(n), x_{2}(n)\right), x_{2}(n)\right)-Q_{2}\left(S_{2}\left(x_{1}(n), x_{2}(n)\right), x_{1}(n)\right)-k 1\right\| \rightarrow 0$. This implies that the infimum of the distances $\| Q_{1}\left(S_{1}\left(x_{1}(n), x_{2}(n)\right), x_{2}(n)\right)-Q_{2}\left(S_{2}\left(x_{1}(n), x_{2}(n)\right), x_{1}(n)\right)-$ $k 1 \|$ on this set is strictly positive: call this $d(\pi, b)$. Note that this function $d(\pi, b)$ is continuous and that the set of permissible $(\pi, b)$ vectors is compact, establishing existence of the claimed positive distance, $d_{\min }>0$.

We finally define the domain $D_{\mu}$ as the set of $\left(x_{1}, x_{2}\right)$ vectors with $\left\|x_{i}\right\| \leq(2 \sqrt{n}+1) R$ and $\min _{k}\left\|x_{1}-x_{2}-k 1\right\| \geq d_{\min }$ such that the population in each of $S_{1}\left(x_{1}, x_{2}\right), S_{2}\left(x_{1}, x_{2}\right)$ is at least $\mu$.

B. Construction of the mapping: Given $\left(x_{1}, x_{2}\right)$, the constituencies of the two institutions are well-defined. They choose policies $\left(x_{1}^{\prime}, x_{2}^{\prime}\right)=\left(Q_{1}\left(S_{1}\left(x_{1}, x_{2}\right), x_{2}\right), Q_{2}\left(S_{2}\left(x_{1}, x_{2}\right), x_{1}\right)\right)$. On our domain, the distinct institutions choose platforms that differ from the 45-degree line by at least $d_{\min }>0$.

However, our mapping is not from $\left(x_{1}, x_{2}\right)$ to $\left(x_{1}^{\prime}, x_{2}^{\prime}\right)$. Instead, we consider the net flow from the two institutions in the move from $\left(x_{1}, x_{2}\right)$ to $\left(x_{1}^{\prime}, x_{2}^{\prime}\right)$. One of the two groups will be a net gainer and the other a net loser. Without loss of generality, let group 1 be the gainer.

In the final mapping, we reduce $x_{1}^{\prime}$ by subtracting a multiple of a unit vector. This reduces the market share going to group 1 while preserving the normal to the dividing hyperplane in the $\Delta^{n-1}$ space. To determine the extent to which we reduce $x_{1}$, define $M_{i}$ as the original population of party $i$ under $\left(x_{1}, x_{2}\right)$, and $M_{i}^{\prime}$ as the population of party $i$ according to $\left(x_{1}^{\prime}, x_{2}^{\prime}\right)$. The definition of $x_{1}^{\prime \prime}$ divides into cases depending on the value of $M_{1}, M_{1}^{\prime}$ and $\lambda \in(0,1)$ as follows:

Case 1 (Interior): If $\mu \leq M_{1}+\lambda\left(M_{1}^{\prime}-M_{1}\right) \leq 1-\mu$, then we define $x_{1}^{\prime \prime}$ by subtracting a multiple of the unit vector from $x_{1}^{\prime}$ until the population in group 1 is exactly $M_{1}+\lambda\left(M_{1}^{\prime}-\right.$ $M_{1}$ ). 
Case 2 (Boundary): If $M_{1}+\lambda\left(M_{1}^{\prime}-M_{1}\right) \geq 1-\mu$, then we define $x_{1}^{\prime \prime}$ by subtracting a multiple of the unit vector from $x_{1}^{\prime}$ until the population in group 1 is exactly $1-\mu .4$

In words, this means that we map from the original $x_{2}$ to the new $x_{2}^{\prime}$, but shift $x_{1}^{\prime}$ to $x_{1}^{\prime \prime} 80$ that the population split according to $\left(x_{1}^{\prime \prime}, x_{2}^{\prime}\right)$ is a weighted average of the original population division and the new population division. To unify the notation, we set $x_{2}^{\prime}=x_{2}^{\prime \prime}$ and map from $\left(x_{1}, x_{2}\right)$ to $\left(x_{1}^{\prime \prime}, x_{2}^{\prime \prime}\right)$.

It follows from A2 and B4 that $S_{i}$ and $Q_{i}\left(S_{i}\left(x_{1}, x_{2}\right), x_{j}\right)$ are continuous in $\left(x_{1}, x_{2}\right)$, so that our mapping to $\left(x_{1}^{\prime \prime}, x_{2}^{\prime \prime}\right)$ will be continuous in $\left(x_{1}, x_{2}\right)$. By subtracting only multiples of the unit vector from $x_{1}^{\prime}$, the minimum distance between $x_{1}^{\prime}-x_{2}^{\prime}$ and the 45-degree line is the same as the distance between $x_{1}^{\prime \prime}-x_{2}^{\prime}$ and the 45-degree line and hence this transformation does not take us out of the domain. Finally, we need to ensure that $\left\|x_{1}^{\prime \prime}\right\| \leq(2 \sqrt{n}+1) R$. Note that the maximum possible utility among $\alpha \in \Delta^{n-1}$ and $x$ in the $R$-ball is $R$ and the minimum possible utility is $-R$. Thus a reduction of $x_{1}^{\prime}$ by $2 R(1,1, \ldots, 1)$ reduces the utility by $2 R$ and hence makes this position less desirable than any possible position within the $R$-ball. Finally, note that since $x_{i}^{\prime}$ was initially inside the $R$-ball, a shift by $2 R$ times the unit vector will keep $x_{i}^{\prime \prime}$ within a $(2 \sqrt{n}+1) R$-ball.

\section{Application of Index Theorem.}

Once again we apply the Lefschetz fixed-point theorem. The domain is bounded, has a smooth boundary and a symmetry property - if $\left(x_{1}, x_{2}\right)$ is in the set so is $\left(x_{2}, x_{1}\right)$. Since $\left\|x_{1}-x_{2}\right\| \geq d_{\text {min }}$, with no additional restrictions, this set would be homeomorphic to $S^{n-1} \times\left\{R^{n}\right\}$. However, for $x_{i}$ in the set we must also eliminate all $x_{j}=x_{i}+\lambda 1$. If we think of this in terms of the possible directions of $\left(x_{1}-x_{2}\right) /\left\|x_{1}-x_{2}\right\|$, then we have eliminated $(1,1, \ldots 1)$ and $(-1,-1, \ldots-1)$. Hence this is like a sphere minus two poles which is homeomorphic to $S^{n-2}$. At this point, the situation is almost identical to the case of Theorem 2 except that we are in one lower dimension. Given that the dimension of $n-1$ is odd (or that the dimension of $n$ is even), the result of the Lefschetz theorem, is that there exists an $\left(x_{1}, x_{2}\right) \in D_{\mu}$ such that $\left(x_{1}, x_{2}\right)=\left(x_{1}^{\prime \prime}, x_{2}^{\prime \prime}\right)$ or $\left(x_{1}, x_{2}\right)=\left(x_{2}^{\prime \prime}, x_{1}^{\prime \prime}\right)$. There exists a pair that either maps back to itself or to its flip. It remains to show that any true fixed-point of this mapping corresponds to an equilibrium and that it is not possible that all fixed points are flip.

D. Existence of Equilibrium. The proof is completed by identical arguments to those used in Theorem 2. There are four distinct types of fixed point: those that involve Case 2 (the boundary fixed points) can be ruled out by Assumption B5 and the fact that $\lambda<1$. Case 1 fixed points are true equilibria. To prove that such a fixed point exists, consider

4 Note that by assumption, group 1 gained population so that $M_{1}+\lambda\left(M_{1}^{\prime}-M_{1}\right) \leq \mu$ is imposible. If group 2 is the one that gains population, then we shift $x_{2}^{\prime}$ in the proscribed monner. 
a sequence of mappings using $\lambda_{n}=\lambda / n$. If all fixed points were antipodal, then the limit of the corresponding sequence of antipodal fixed points would be a point with an equal population division which maps to the reverse normal, contradicting B6. Q.E.D.

Once again, changing the proof to cover the case of nonviable small institutions [B5'] is straightforward; we simply shift the direction of the mapping, replacing $\lambda$ by $-\lambda$.

TheOREM (LefSChETZ FIXED-PONNT THEOREM, (Munkres, 1984)): Let $K$ be a finite complex; let $F:|K| \mapsto|K|$ be a continuous map. If the Lefschetz number, $\Lambda(F) \neq 0$, then $F$ has a fixed point.

Theorem 4 (EXISTENCE OF FIXED POINT OR ANTIPODE, (MUNKRes)): Let $\pi \epsilon$ $S^{n-1}$. (a) Suppose that to each such $\pi$ we associate a non-trivial interval $[f(\pi), g(\pi)]$ on the real line. $(\mathrm{b})$ Suppose further that $f$ and $g$ are continuous functions of $\pi$ and (c) that $f(-\pi)=-g(\pi)$. Let $\Omega \subset S^{n-1} \times R$ be the space of all pairs $(\pi, b)$ with $f(\pi) \leq b \leq g(\pi)$.

Under these hypothesis, for $n$ odd, it follows that if $F: \Omega \mapsto \Omega$ is a continuous function, then there is either a fixed point or an antipodal fixed point: there exists a $(\pi, b) \in X$ such that $F(\pi, b)=(\pi, b)$ or $F(\pi, b)=(-\pi,-b)$.

ProOF: The theorem is a consequence of the Lefschetz fixed-point theorem. Under hypothesis hypothesis (a) and (b), the space $\Omega$ is homeomorphic to $S^{n-1} \times[0,1]$, so it is triangulable and compact. Thus the hypothesis of the Lefschetz theorem are satisfied. For any continuous map $F: \Omega \mapsto \Omega$ there is an associated integer $d$ (which we call the degree of $F$ ) determined by the homeomorphism $F_{*}: H_{n-1}(X) \mapsto H_{n-1}(X)$ in homology. The Lefschetz number $\Lambda(F)$ of $F$ is $1+(-1)^{n-1} d(F)$, where $d(F)$ is the "degree" of $F$.

Next, we introduce the continuous map $A: \Omega \mapsto \Omega$ given by $a(\pi, b)=(-\pi,-b)$. This map takes each point to its antipode. Since it is fixed-point free, $\Lambda(a)=1+(-1)^{n-1} d(A)=$ 0 , so its degree must be $(-1)^{n}$.

If the theorem were false then both $F$ and $a \circ F$ must be fixed-point free. $\Lambda(F)=0$ implies that the degree of $F$ is $(-1)^{n} . \Lambda(a \circ F)=0$ implies that the degree of $F$ is 1 :

$$
1+(-1)^{n-1} d(A \circ F)=1+(-1)^{n-1}\left((-1)^{n} d(F)\right)=1-d(F)=0
$$

since degree is multiplicative. This leads to a contradiction whenever $(-1)^{n} \neq 1$ or $n$ is odd.

Q.E.D.

The theorem applies directly to our membership-based framework since $D_{\mu}$ satisfies (a), (b), and (c) and the mapping from $(\pi, b)$ to $\left(\pi^{\prime}, b^{\prime \prime \prime}\right)$ is continuous.

5 By non-trivial, we mean $f(\pi)<g(\pi)$. 